\title{
Early intervention in pulmonary arterial hypertension associated with systemic sclerosis: an essential component of disease management
}

\author{
E. Hachulla* and C.P. Denton\#
}

ABSTRACT: Pulmonary arterial hypertension (PAH) is a life-threatening complication of systemic sclerosis (SSc). However, PAH-specific treatments are available and can significantly improve survival of patients, especially those diagnosed in World Health Organization (WHO) functional class (FC) II. Registry data have shown that without screening, more than two-thirds of PAH-SSc patients are in WHO FC III or IV when diagnosed. The recognised predisposition of SSc patients to develop PAH should mean that an optimal screening programme will enable the early diagnosis of $\mathrm{PAH}$, and provide the opportunity for earlier treatment, in this population. Evidence-based treatment guidelines advocate the use of oral PAH-specific therapies, including bosentan, ambrisentan, sildenafil (I-A recommendation), tadalafil (I-B recommendation) and sitaxentan (IIA-C recommendation), for patients in WHO FC II. A randomised, placebo-controlled trial of bosentan in WHO FC II PAH patients, including cases of PAH-SSc, showed improved haemodynamics in actively treated patients and a reduced risk of progression from WHO FC II to FC III. For PAH patients diagnosed in WHO FC III, the treatment goal is to improve to WHO FC II. Data from bosentan trials have shown that nearly one-quarter of patients in WHO FC III at baseline can attain WHO FC II status with monotherapy. Maintenance of PAH-SSc patients in WHO FC II with monotherapy is unrealistic, and sequential goal-directed combination therapy is now becoming an accepted treatment strategy. It is hoped that earlier diagnosis, coupled both with regular assessments to ensure treatment goals are being met and timely, appropriate treatment, will further improve the survival rates of those PAH-SSc patients.

KEYWORDS: Early intervention, pulmonary arterial hypertension, systemic sclerosis, World Health Organization functional class II

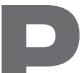

ulmonary arterial hypertension (PAH) is a progressive disease with an extremely poor prognosis. PAH associated with systemic sclerosis (PAH-SSc) is particularly aggressive, accounting for $30 \%$ of deaths among SSc patients [1]; if left untreated, PAH-SSc is associated with a median survival time of $1 \mathrm{yr}$ following diagnosis [2, 3]. This is much lower than the corresponding predicted survival rate in patients with idiopathic pulmonary arterial hypertension (IPAH) [3, 4]. Recent advances in diagnostic techniques and the emergence of evidence-based therapies for $\mathrm{PAH}$ facilitate earlier detection of the disease, thereby permitting earlier initiation of effective treatment. Early detection of PAH and prompt initiation of effective therapy is considered an essential component of disease management, as patients diagnosed earlier in the course of the disease derive a more pronounced benefit from therapy $[5,6]$.

\section{WORLD HEALTH ORGANIZATION FUNCTIONAL CLASS II STATUS: AN IMPORTANT CLINICAL TARGET}

The severity of $\mathrm{PAH}$, including $\mathrm{PAH}$ in SSc patients can be classified clinically using the World Health Organization (WHO) functional classification system [7]. Two decades ago, historical data demonstrated that the median survival of untreated patients with IPAH or
AFFILIATIONS

${ }^{*}$ Claude Huriez Hospital, Lille, France.

${ }^{\#}$ Royal Free Campus, University College Medical School, London, UK.

CORRESPONDENCE

E. Hachulla

Dept of Internal Medicine

National Reference Center for

Scleroderma

Claude Huriez Hospital

University of Lille

59037 Lille cedex

France

E-mail: ehachulla@chru-lille.fr

Received:

Aug 242010

Accepted after revision:

Sept 302010

PROVENANCE

Publication of this peer-reviewed article was supported by Actelion Pharmaceuticals Ltd, Switzerland (unrestricted grant, European

Respiratory Review issue 118).

European Respiratory Review Print ISSN 0905-9180

Online ISSN 1600-0617 
heritable PAH was 6 months, 2.5 yrs and 6 yrs for patients in WHO functional class (FC) IV, III and II, respectively [8]. Survival of patients diagnosed in FC II is superior, partly because the disease is less severe at this stage (lead time bias). However, other factors, such as individual or genetic susceptibility, may explain why some patients with less severe disease at diagnosis have a better prognosis. $\mathrm{PAH}$ is a progressive disease even in mildly symptomatic patients $[9,10]$ and, over time, pulmonary arterial pressure $(\mathrm{Ppa})$ and pulmonary vascular resistance (PVR) increase and cardiac index $(\mathrm{CI})$ decreases, leading to clinical deterioration and, ultimately, right heart failure. Recently published European Society of Cardiology (ESC)/European Respiratory Society (ERS) guidelines concluded that WHO FC II status represents an important threshold indicative of better prognosis [11], making maintenance or attainment of FC I or FC II a key goal of therapy in the management of patients with $\mathrm{PAH}$, including those with PAH-SSc. In the context of PAH-SSc, two key questions need to be addressed: 1) how feasible is it to identify PAH-SSc patients earlier in the course of their disease; and 2) once identified, is earlier treatment of these patients associated with significant clinical benefit?

\section{Timely diagnosis of PAH in SSc patients is currently the greatest challenge for physicians}

Pulmonary complications contribute to most deaths in SSc patients, and the greatest current challenge for physicians is the early diagnosis and effective treatment of such complications. In the current treatment era, the 3-yr survival rate of PAH-SSc patients in FC II at diagnosis is in the region of $70-75 \%$ (fig. 1) $[12,13]$. This means that one-quarter of patients are likely to die within 3 yrs of diagnosis, a rate which is higher than for some cancers. Critically, only $16.7 \%$ of PAH-SSc patients are in FC II at diagnosis [13], meaning that $>80 \%$ of PAH-SSc patients have a 3-yr life expectancy of $20-70 \%$ by the time they are diagnosed $[12,13]$. PAH-targeted therapies are of therapeutic benefit in patients with more advanced disease in WHO FC III and IV. However, earlier diagnosis should allow these therapies to be used to their full advantage, and could help improve outcomes for PAH-SSc patients.

\section{Establishing effective screening protocols for SSc patients}

A consensus statement, published by American pulmonary hypertension $(\mathrm{PH})$ physicians [14], recommended yearly echocardiographic screening of SSc patients. However, in evidence-based guidelines produced by the ESC/ERS [11], the data were not deemed sufficiently strong to recommend yearly echocardiograms for all SSc patients; instead, these guidelines advocate annual echocardiographic screening in symptomatic SSc patients and its consideration in asymptomatic patients [11]. Since SSc patients are chronically ill, the problem of identifying which symptoms should lead to a screening test is a difficult one that has yet to be resolved. In reality, most SSc patients will display some symptoms, such as dyspnoea, fatigue, weakness or abdominal distension, making the decision of when to re-evaluate the patient of prime importance, rather than whether to perform an initial echocardiogram. Because some of them may be at an early stage of $\mathrm{PAH}$ and therefore asymptomatic, the true incidence of PAH among SSc patients remains unknown, but is at least 0.61 per 100 patient-yrs [15].

\section{Echocardiographic assessment of PAH}

During the course of an echocardiogram, there are a number of different measurements that can be taken as an indication of the presence of PH. The ESC/ERS diagnostic algorithm in SSc patients with suspected $\mathrm{PAH}$ is based on the maximal tricuspid regurgitation velocity (TRV) [11].

1) A patient with a TRV of $\leqslant 2.8 \mathrm{~m} \cdot \mathrm{s}^{-1}$ or pulmonary artery systolic pressure (Ppa,syst) of $\leqslant 36 \mathrm{mmHg}$ (assuming mean right atrial pressure is $\leqslant 5 \mathrm{mmHg}$ ) is unlikely to have $\mathrm{PH}$, and right heart catheterisation (RHC) based on these measurements is not justified.

2) At the other end of the scale, a patient with a TRV of $>3.4 \mathrm{~m} \cdot \mathrm{s}^{-1}$ or a $P$ pa,syst of $>50 \mathrm{mmHg}$ is likely to have $\mathrm{PH}$, and RHC is indicated in order to confirm the diagnosis.

3) In patients with a TRV between these extremes (i.e. 2.9 $3.4 \mathrm{~m} \cdot \mathrm{s}^{-1}$ ), other additional indicators of $\mathrm{PH}$ should be considered and clinical judgment is required as to whether RHC is warranted. In such cases, it is up to the treating physician, working alongside the echocardiography department, to make the decision.

This algorithm is relatively easy to employ and, using a slightly adapted version (fig. 2), it has been possible to detect $\mathrm{PAH}$ in SSc patients following RHC dependent upon echocardiographic readings and the presence or absence of dyspnoea. Importantly, by using this adapted algorithm, $56.3 \%$ of patients were diagnosed in FC II $[16,17]$. In SSc dyspnoea is a common symptom and differential diagnosis is important. As well as parenchymal lung disease or $\mathrm{PH}$ the possibilities of anaemia, left ventricular dysfunction, pleuro-pericardial disease, musculoskeletal compromise and physical deconditioning should also be considered. Minimal evaluation should include investigations that address these possibilities including chest radiography, routine blood assessment, ECG, and thorough clinical examination as well as echocardiography and pulmonary function testing.

There are alternative structural and functional echocardiographic features that could be used to indicate diagnostic RHC. Structurally, an enlarged right ventricle $\left(>12-13 \mathrm{~cm}^{2}\right)$, even in the absence of an elevated TRV, could indicate PH, although there is little consensus amongst echocardiographers.

Additional clinical information that may also be indicative of $\mathrm{PH}$ is a diffusing lung capacity of the lung for carbon monoxide of $<60 \%$ in the absence of extensive interstitial lung disease (i.e. disease extent on high-resolution computed tomography $>20 \%$ and/or forced vital capacity $<70 \%$ [18]). N-terminal pro-brain natriuretic peptide (NT-proBNP) levels are also significantly correlated with haemodynamics [16]; even with a normal echocardiogram, an NT-proBNP level more than three times the upper limit of normal is highly suggestive of $\mathrm{PH}$. Elevated NT-proBNP levels may be predictive of PAH development in SSc patients [19], and as NT-proBNP detection is simple and non-invasive, it may be an attractive screening tool to be used alongside other parameters [20].

Although ECG is performed routinely in assessing patients at risk of developing $\mathrm{PH}$, its utility is limited as a screening tool since abnormalities are not specific and are more likely to reflect established $\mathrm{PH}$. However, it is valuable to help define 

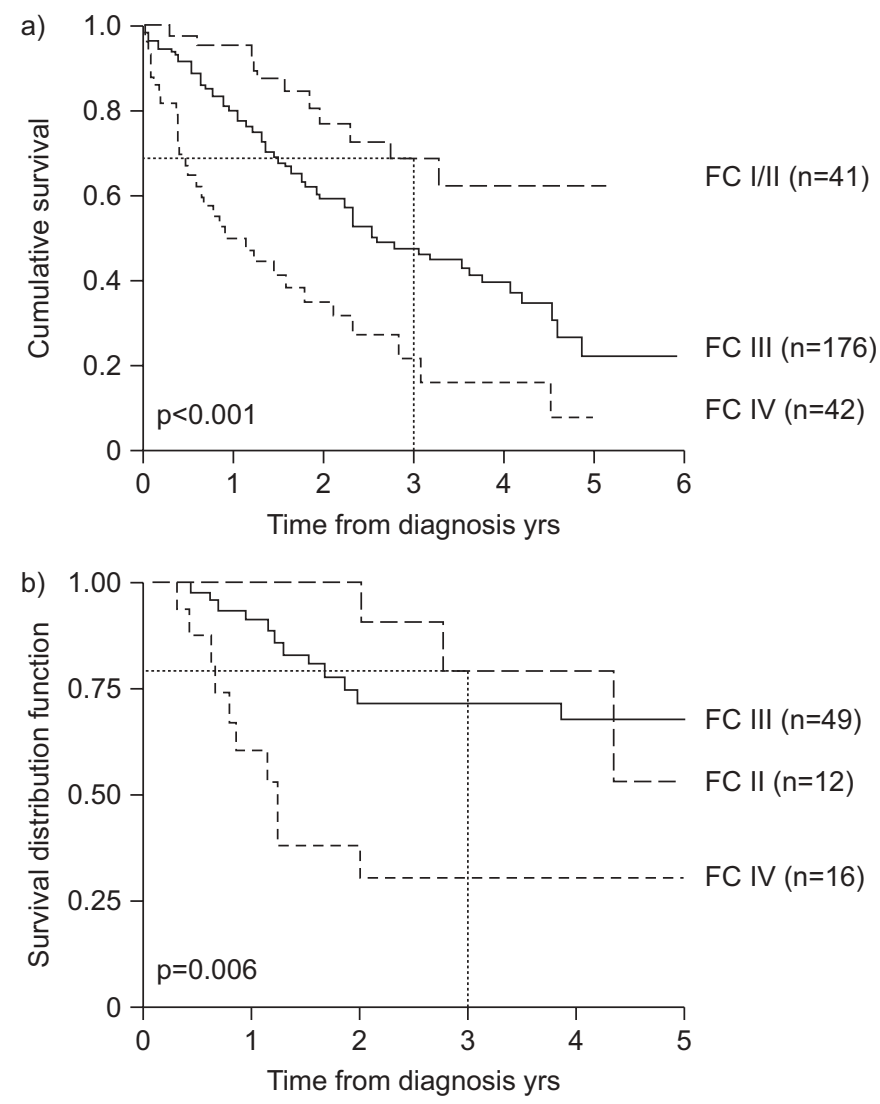

FIGURE 1. Survival of pulmonary arterial hypertension associated with systemic sclerosis (PAH-SSc) patients according to World Health Organization functional class (FC) at diagnosis. a) The UK National registry of all incident cases of PAH-SSc. Reproduced from [12] with permission from the publisher. b) All incident cases of PAH-SSc seen after 2000 ( $n=78$; Béclère, Lille, Cochin). Reproduced from [13] with permission from the publisher.

other cardiac pathology and should be included in assessment schedules.

The ongoing DETECT study [21] aims to refine the screening process in SSc patients in order to diagnose $\mathrm{PAH}$ earlier and with greater accuracy. DETECT will compare the discriminatory and predictive value of a number of potential screening tools, both alone and in combination, including NT-proBNP, ECG and echocardiography. The study will be performed over a 3-yr period, and it is hoped that the findings of the study will inform future guidelines.

Returning to the first of the questions posed earlier, it is clearly possible to diagnose many cases of PAH-SSc when the patients are in FC II. Given this situation, it is logical to ask the second question: what evidence is available that treatment of patients in FC II is associated with significant clinical benefit?

\section{PAH-SPECIFIC THERAPIES FOR FC II ATTAINMENT AND MAINTENANCE}

The feasibility of earlier diagnosis of PAH-SSc brings with it the responsibility of appropriate early treatment. Patients in WHO FC II should be treated with PAH-specific therapies, in accordance with the recently published treatment algorithm [22]. This algorithm recommends first-line use of the PAH-specific therapies

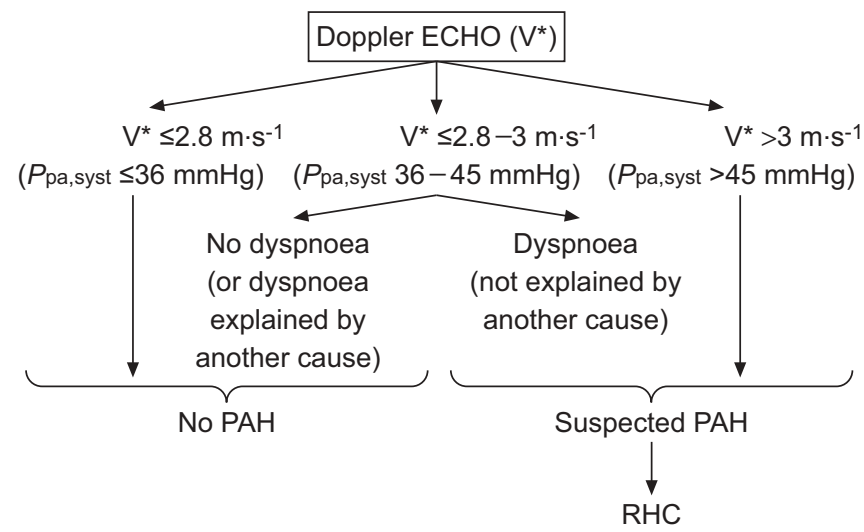

FIGURE 2. Screening protocol for systemic sclerosis patients. V*: systolic regurgitant tricuspid flow velocity; $P$ pa,syst: systolic pulmonary artery pressure; $\mathrm{PAH}$ : pulmonary arterial hypertension; $\mathrm{RHC}$ : right heart catheterisation. Reproduced from [15] with permission from the publisher.

bosentan, ambrisentan, sitaxentan, tadalafil and sildenafil in FC II $\mathrm{PAH}$ patients, including those with PAH-SSc, based on robust evidence derived from well-designed, randomised controlled trials. Acute vasoreactivity testing of IPAH patients at diagnosis is also recommended, with subsequent administration of calciumchannel blockers in those found to be acutely vasoreactive. This approach is not recommended for patients with $\mathrm{PAH}$ associated with connective tissue diseases (CTD), however, as these patients do not maintain vasoreactivity in the long term [23]. RHC-based diagnosis of $\mathrm{PAH}$ is important as it permits the potentially important cases of pulmonary venous hypertension to be identified robustly. In addition, the possibility of pulmonary venoocclusive disease being a contributory factor must be considered [24], as cases with associated venous involvement may respond adversely to PAH-specific therapies.

The value of early therapeutic intervention in PAH was unequivocally demonstrated in the EARLY trial. This 6-month study remains the only randomised, placebo-controlled trial dedicated to FC II patients, and included some cases of PAH-SSc [10]. EARLY was designed to gain further insight into early stage disease and to investigate the effects of the dual endothelin-receptor antagonist (ERA) bosentan specifically in FC II patients. Inclusion of a placebo arm provided compelling evidence on the rapid progression of PAH in the absence of therapy, even in mildly symptomatic patients. The co-primary end-points of the EARLY trial were changes in PVR and exercise capacity, as measured by the 6-min walk distance (6MWD). Disease progression was assessed by the secondary end-points of time to clinical worsening (TTCW) and change in WHO FC.

A highly significant reduction in PVR of $22.6 \%$ compared with placebo $(p<0.0001)$, and a trend toward improvement in $6 \mathrm{MWD}(\mathrm{p}=0.076)$, were observed after 6 months of treatment with bosentan (fig. 3). The absence of a significant improvement in 6MWD with bosentan may reflect the fact that enrolled patients had a relatively well-preserved exercise capacity, which may be difficult to improve. A significant delay in PAH progression was seen in patients treated with bosentan compared with placebo. A clinical worsening event was experienced by $14 \%$ of placebo-treated patients, compared 

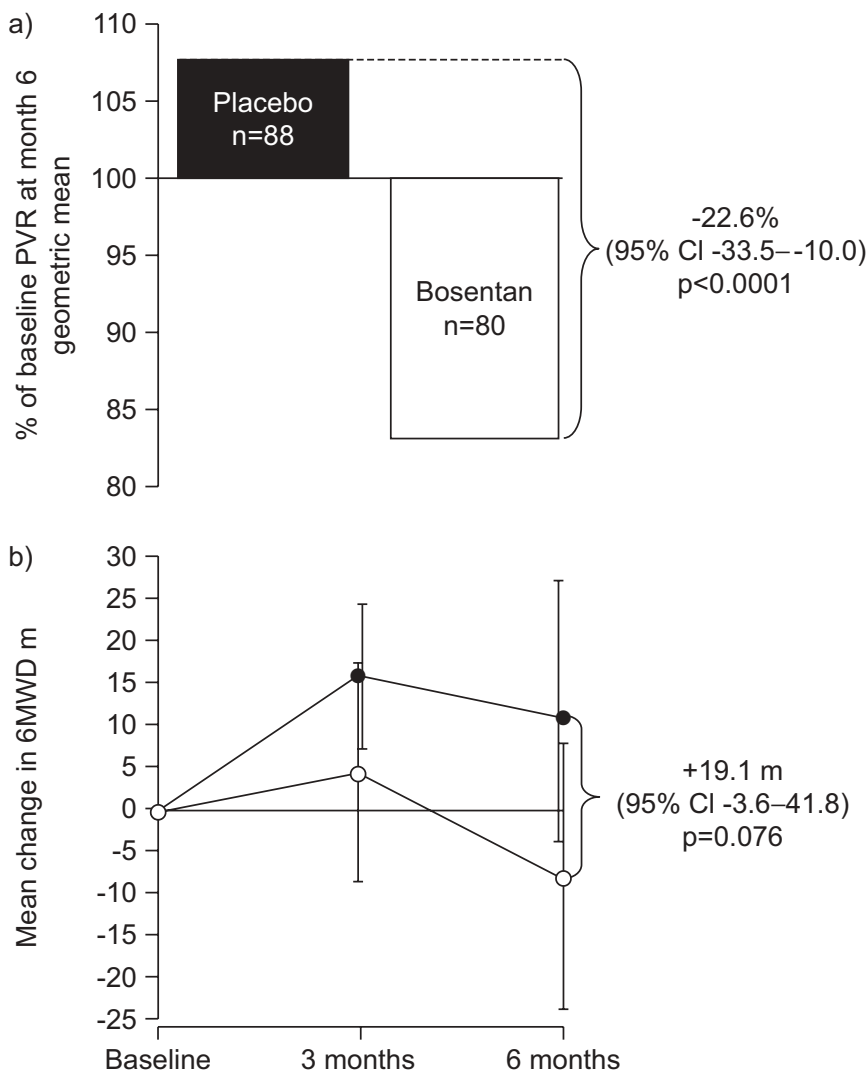

FIGURE 3. EARLY co-primary end-points. a) Effect of bosentan on pulmonary vascular resistance (PVR) after 6 months. b) Effect of bosentan ( $; n=86$ ) on 6-min walk distance (6MWD) after 6 months. $\bigcirc$ : placebo $(n=91)$. Reproduced from [10] with permission from the publisher.

with only $3 \%$ of those treated with bosentan, representing a relative risk reduction of $77 \%$ for bosentan-treated patients $(\mathrm{p}=0.0114)$.

Importantly with respect to current discussion on the importance of FC II status, significantly fewer patients in the bosentan $(3 \%)$ group deteriorated from FC II to FC III or IV after 6 months of therapy, compared with placebo-treated patients $(13 \%)$, representing a relative risk reduction of $77 \%$ $(p=0.0285)$ (fig. 4). The pronounced effect of bosentan on TTCW and the associated improvement or stabilisation in WHO FC reinforce the delay in disease progression observed with bosentan, and suggest that intervention when patients are in FC II can alter the progression of PAH.

Significant reductions in NT-proBNP levels and improvements in the short form-36 health transition index were also observed in bosentan-treated patients relative to the placebo group. A subgroup of patients who received concomitant sildenafil showed improvements consistent with the overall results. The safety and tolerability profile of bosentan was consistent with that observed in previous placebo-controlled clinical trials [25, 26]. An open-label extension of EARLY is ongoing to establish the impact of early intervention on long-term outcome in $\mathrm{PAH}$.

Maintenance and attainment of FC II in PAH-SSc patients $17 \%$ of the patients enrolled in the EARLY trial had PAH associated with CTD, including SSc. However, it is important

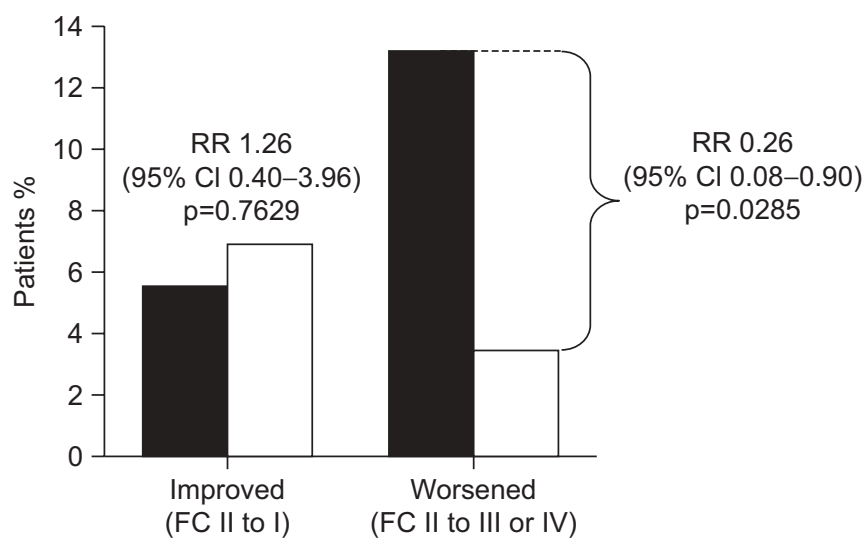

FIGURE 4. Effect of bosentan on World Health Organization functional class (WHO FC). In EARLY, after 6 months treatment significantly fewer patients in the bosentan group ( $\square ; n=87$ ) showed deterioration in WHO FC, compared with those on placebo $(\boldsymbol{\square} ; n=91)$. Reproduced from [10] with permission from the publisher

not to assume that the results of the whole patient population, consisting primarily of IPAH patients, apply equally to a subgroup of those with PAH-SSc. In support of the EARLY data, a prospective study of $57 \mathrm{PAH}-\mathrm{SSc}$ patients in FC II at the Royal Free Hospital in London, UK, indicated that survival in $\mathrm{PAH}-\mathrm{SSc}$ has improved since the introduction of ERAs [27].

Although echocardiographic screening of SSc patients and subsequent RHC is able to diagnose a significant proportion of PAH-SSc patients in FC II, almost one-half will have more advanced disease at diagnosis [17, 28]. For these patients, achieving an improvement in WHO FC is a key therapeutic aim. Evaluation of the effect of bosentan in the 66 patients with PAH-SSc enrolled in the pivotal trials (BREATHE (Bosentan Randomized Trial of Endothelin Antagonist Therapy)-1 [26] and study 351 [25]) and their open-label extensions [29] demonstrated that, of the 40 patients on bosentan monotherapy, $25 \%$ improved in FC. Since $95.5 \%$ of patients were in FC III at baseline, this effectively means that close to one-quarter attained at least FC II status.

In a further study, 53 patients with PAH related to CTD (70\% with limited $(55 \%)$ or diffuse $(25 \%)$ SSc) in FC III received bosentan for 48 weeks. After 48 weeks, FC improved in $27 \%$ of patients (95\% CI 16-42\%), was unchanged in 57\% (95\% CI $42-$ $71 \%$ ) and worsened in 16\% (95\% CI 7-29\%) (fig. 5) [30].

Definitive diagnosis of PAH can only be achieved with RHC. However, RHC is an invasive procedure, and it is impractical to subject all SSc patients to regular catheterisation to assess right heart function and diagnose PAH early. In IPAH, the insidious onset of nonspecific symptoms, such as increasing exercise intolerance, means that diagnosis is often made late, after more likely diseases have been discounted. However, the prevalence of haemodynamically proven $\mathrm{PAH}$ in large SSc patient cohorts is $7-12 \%[16,28,31]$, meaning that a recognised predisposition exists for development of PAH in SSc patients. With regular screening, therefore, the early diagnosis of $\mathrm{PAH}$ is a realistic possibility in this patient population. Without screening, data from the French national registry have shown that the majority of patients are diagnosed in FC III or IV, and as such are already severely comprised [32]. Diagnosis of PAH 


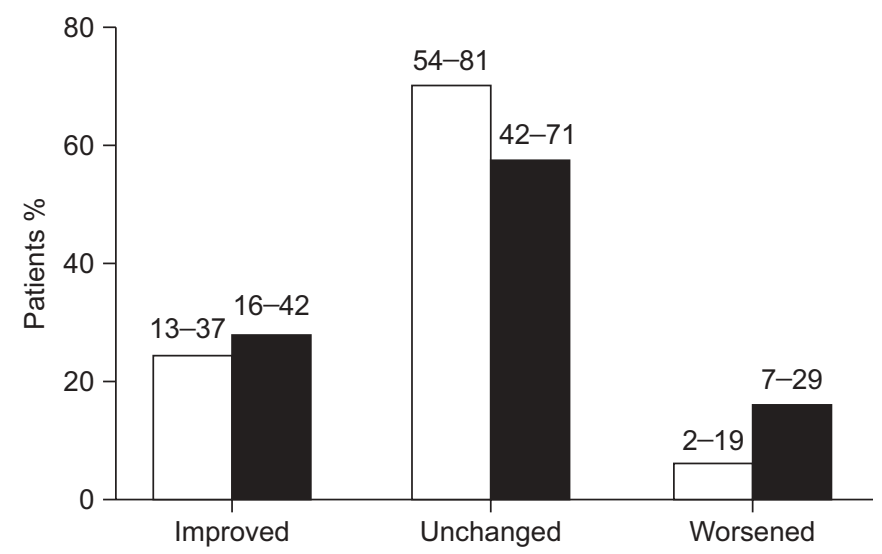

FIGURE 5. Improvement/worsening in World Health Organization functional class (WHO FC) at week $16(\square)$ and week 48 (汭. Two patients at week 16 and three at week 48 were discontinued because of pulmonary arterial hypertension worsening/death and were assigned WHO FC IV at week 16 and week 48, respectively, as per protocol. $n=51.95 \%$ confidence intervals are indicated. Reproduced from [30] with permission from the publisher

in FC I or II is challenging; even when patients begin to deteriorate, symptoms are nonspecific and patients can be reluctant to present to their physician [17]. Therefore, despite the potential for earlier diagnosis compared with the IPAH patient population, PAH-SSc is still frequently identified late, with more than two-thirds of patients in FC III or IV at the time of diagnosis [12, 15, 33].

Sildenafil has also been shown to result in improvements in WHO FC among patients with PAH associated with CTD [34]. Following 12 weeks of treatment, improvement of at least one WHO FC was observed in six (29\%), eight $(40 \%)$ and eight (42\%) patients receiving 20, 40 and $80 \mathrm{mg}$ sildenafil, respectively, compared with one (5\%) patient receiving placebo. As all of the CTD-PAH patients enrolled in the study were in WHO FC II/III, except one patient in FC IV in the sildenafil group, the results suggest that overall $\sim 38 \%$ achieved $\mathrm{WHO}$ FC II status with sildenafil therapy. However, the number of cases is too small for a robust conclusion to be drawn. It should also be noted that the approved dose for sildenafil in $\mathrm{PAH}$ is $20 \mathrm{mg}$. No significant differences in WHO FC were observed with sitaxentan compared with placebo in a post hoc analysis of PAH-CTD patients in STRIDE (Sitaxsentan To Relieve Impaired Exercise) [35].

\section{Appropriate treatment targets for patients with PAH-SSc}

The ESC/ERS guidelines [11] identified a number of parameters for assessing disease severity, stability and prognosis in $\mathrm{PAH}$. As well as maintenance in FC II (or FC I if possible), discussed in detail above, absence of right ventricular failure, slow disease progression, absence of syncope, 6MWD $>500 \mathrm{~m}$, peak oxygen uptake $>15 \mathrm{~mL} \cdot \mathrm{kg}^{-1} \cdot \mathrm{min}^{-1}$, normal or near-normal NT-proBNP, tricuspid annular plane systolic excursion $>2.0 \mathrm{~cm}$, right atrial pressure $<8 \mathrm{mmHg}$ and cardiac index $\geqslant 2 \mathrm{~L} \cdot \mathrm{min}^{-1} \cdot \mathrm{m}^{-2}$ have all been associated with better outcomes. However, these values are derived largely from data in IPAH patients and some of these targets, such as a $6 \mathrm{MWD}>500 \mathrm{~m}$, may not always be achievable in the PAH-SSc patient population; PAH may be just one of several factors contributing to limited exercise capacity, including interstitial lung disease and musculoskeletal involvement [36]. Therefore, other treatment goals are required to evaluate disease severity and to monitor the impact of therapy in these patients.

If a PAH-SSc patient is still in FC I/II after 4 months of treatment, prognosis is relatively good compared with patients who have deteriorated to FC III/IV at this time $(p=0.007)$ [37]. Therefore, patients should be reviewed after 4 months and if FC status is not favourable, additional therapy should be considered. Cardiac index also has a good prognostic value. A cardiac index $>2.71 \mathrm{~L} \cdot \mathrm{min}^{-1} \cdot \mathrm{m}^{-2}$ is associated with a better prognosis than a cardiac index of $<2.71 \mathrm{~L} \cdot \mathrm{min}^{-1} \cdot \mathrm{m}^{-2}(\mathrm{p}=0.03)$ [37].

Maintenance of PAH-SSc patients in WHO FC II with longterm monotherapy is unrealistic, and the elucidation of three key pathological pathways in $\mathrm{PAH}$ makes combination therapy an attractive treatment option [38]. Combination therapy is an accepted approach in other serious, chronic diseases, such as HIV infection, congestive heart failure and cancer, and combination therapy is included in the recently published $\mathrm{PH}$ treatment guidelines [11]. A number of studies have demonstrated the benefits of combination therapy in the management of PAH secondary to a variety of aetiologies [39-41]. Unpublished observations from the Royal Free Hospital SSc patient cohort suggest that combination therapy is effective in patients with PAH-SSc: 3-yr mortality rates of 45\%, $55 \%$ and $69 \%$ were reported prior to the availability of oral therapies, with the advent of first-line ERAs and with use of goal-directed combination therapy, respectively.

\section{ASSESSING LONG-TERM OUTCOMES IN PAH-SSC}

Short-term targets, such as FC II maintenance after 4-6 months of therapy, are valuable markers of response to therapy and influence patient management. However, the real aim of therapy is to improve long-term outcomes and increase survival in PAH-SSc patients. Although meta-analysis of the IPAH population has demonstrated improved survival among patients treated with targeted therapies, improvements are still needed and mortality remains high [42].

As discussed above, earlier diagnosis of PAH-SSc is facilitated by a comprehensive screening programme that focuses on noninvasive tests with confirmatory RHC in cases of suspected $\mathrm{PAH}$, which allows the earlier treatment of patients with PAHtargeted therapies. Kaplan-Meier estimates of survival in the prevalent CTD-PAH cohort of the two pivotal bosentan trials and their open-label extensions were $85.9 \%$ and $73.4 \%$ after 1 and 2 yrs, respectively [27], which compares favourably with historical data $[2-4,43]$. The hardest end-point available is survival, and survival is still a meaningful end-point given the very high mortality rate associated with PAH-SSc. Maintenance of PVR <650 dyn appears to be associated with substantially more favourable survival, demonstrating the importance of influencing haemodynamics to achieve a positive impact on long-term outcome.

If, as is hoped, survival rates improve in PAH-SSc as more patients are diagnosed and treated earlier in the course of their disease, alternative end-points, such as TTCW, become more important as measures of long-term outcomes. However, TTCW needs to be validated in the very challenging SSc 
patient population; musculoskeletal involvement in SSc has a greater impact on measurements of exercise capacity compared with severity of PAH and a number of comorbidities could contribute to breathlessness, such as lung involvement and cardiac involvement. In addition, there are multiple causes of disease-related death which are not necessarily a direct result of $\mathrm{PAH}$.

\section{CONCLUSION}

Even in mildly symptomatic patients, $\mathrm{PAH}$ is rapidly progressive and, if untreated, $13 \%$ of patients in WHO FC II at diagnosis will deteriorate to WHO FC III/IV within 6 months [9]. Early intervention with bosentan in WHO FC II $\mathrm{PAH}$ patients can significantly delay $\mathrm{PAH}$ progression and may alter the natural course of the disease.

Effective screening of SSc patients can enable earlier diagnosis of PAH-SSc, so that these patients can benefit from earlier intervention with targeted $\mathrm{PAH}$ therapies, according to the published guidelines [11]. Earlier treatment is especially critical in the PAH-SSc patient population, where the disease is particularly aggressive. Appropriate treatment goals have to be defined and patients should be re-evaluated after 4-6 months of first-line treatment or changes in PAH-specific therapy.

\section{STATEMENT OF INTEREST}

E. Hachulla has received less than $€ 10,000$ from Actelion, Pfizer and GSK for attending symposia, speaking and consultant work. C.P. Denton has received research grant funding and acted as a consultant to Actelion Pharmaceuticals, Pfizer and GlaxoSmithKline. He has also received lecture fees from these companies.

\section{ACKNOWLEDGEMENTS}

We received editorial assistance from J. Heagerty, Elements Communications Ltd (Westerham, UK), supported by Actelion Pharmaceuticals Ltd (Allschwil, Switzerland).

\section{REFERENCES}

1 Steen VD, Medsger TA. Changes in causes of death in systemic sclerosis, 1972-2002. Ann Rheum Dis 2007; 66: 940-944.

2 Koh ET, Lee P, Gladman DD, et al. Pulmonary hypertension in systemic sclerosis: an analysis of 17 patients. Br J Rheumatol 1996; 35: 989-993.

3 Kawut SM, Taichman DB, Archer-Chicko CL, et al. Hemodynamics and survival in patients with pulmonary arterial hypertension related to systemic sclerosis. Chest 2003; 123: 344-350.

4 Humbert M, Sitbon O, Yaici A, et al. Survival in incident and prevalent cohorts of patients with pulmonary arterial hypertension. Eur Respir J 2010; 36: 549-555.

5 McLaughlin VV, Shillington A, Rich S. Survival in primary pulmonary hypertension: the impact of epoprostenol therapy. Circulation 2002; 106: 1477-1482.

6 Sitbon O, Humbert M, Nunes $\mathrm{H}$, et al. Long-term intravenous epoprostenol infusion in primary pulmonary hypertension: prognostic factors and survival. J Am Coll Cardiol 2002; 40: 780-788.

7 Barst RJ, McGoon M, Torbicki A, et al. Diagnosis and differential assessment of pulmonary arterial hypertension. J Am Coll Cardiol 2004; 43: S40-S47.

8 D'Alonzo GE, Barst RJ, Ayres SM, et al. Survival in patients with primary pulmonary hypertension. Results from a national prospective registry. Ann Intern Med 1991; 115: 343-349.
9 Brenot F. Primary pulmonary hypertension. Case series from France. Chest 1994; 105: Suppl. 2, 33S-36S.

10 Galiè N, Rubin LJ, Hoeper M, et al. Treatment of patients with mildly symptomatic pulmonary arterial hypertension with bosentan (EARLY study): a double-blind, randomised controlled trial. Lancet 2008; 371: 2093-2100.

11 Galiè N, Hoeper MM, Humbert M, et al. The Task Force for Diagnosis and Treatment of Pulmonary Hypertension of European Society of Cardiology (ESC); European Respiratory Society (ERS); International Society of Heart and Lung Transplantation (ISHLT). Guidelines for the diagnosis and treatment of pulmonary hypertension. Eur Respir J 2009; 34: 1219-1263.

12 Condliffe R, Kiely DG, Peacock AJ, et al. Connective tissue diseaseassociated pulmonary arterial hypertension in the modern treatment era. Am J Respir Crit Care Med 2009; 179: 151-157.

13 Hachulla E, Launay D, Yaici A, et al. Pulmonary arterial hypertension associated with systemic sclerosis in patients with functional class II dyspnoea: mild symptoms but severe outcome. Rheumatology 2010; 49: 940-944.

14 McLaughlin VV, Archer SL, Badesch DB, et al. ACCF/AHA 2009 expert consensus document on pulmonary hypertension a report of the American College of Cardiology Foundation Task Force on Expert Consensus Documents and the American Heart Association developed in collaboration with the American College of Chest Physicians; American Thoracic Society, Inc.; and the Pulmonary Hypertension Association. J Am Coll Cardiol 2009; 53: 1573-1619.

15 Hachulla E, de Groote P, Gressin V, et al. The three-year incidence of pulmonary arterial hypertension associated with systemic sclerosis in a multicenter nationwide longitudinal study in France. Arthritis Rheum 2009; 60: 1831-1839.

16 Mukerjee D, Yap LB, Holmes AM, et al. Significance of plasma Nterminal pro-brain natriuretic peptide in patients with systemic sclerosis-related pulmonary arterial hypertension. Respir Med 2003; 97: 1230-1236.

17 Levine DJ. Diagnosis and management of pulmonary arterial hypertension: implications for respiratory care. Respir Care 2006; 51: 368-381.

18 Goh NS, Desai SR, Veeraraghavan S, et al. Interstitial lung disease in systemic sclerosis: a simple staging system. Am J Respir Crit Care Med 2008; 177: 1248-1254.

19 Allanore Y, Borderie D, Avouac J, et al. High N-terminal pro-brain natriuretic peptide levels and low diffusing capacity for carbon monoxide as independent predictors of the occurrence of precapillary pulmonary arterial hypertension in patients with systemic sclerosis. Arthritis Rheum 2008; 58: 284-291.

20 Vachiéry J-L, Coghlan G. Screening for pulmonary arterial hypertension in systemic sclerosis. Eur Respir Rev 2009; 18: 162-169.

21 Vonk M, Coghlan G, Bonderman D, et al. The DETECT study: A two-stage, prospective, observational, cohort study in scleroderma patients to evaluate screening tests and the incidence of pulmonary arterial hypertension and pulmonary hypertension. Clin Exp Rheumatol 2010; 28: Suppl., 55.

22 Barst RJ, Gibbs JS, Ghofrani HA, et al. Updated evidence-based treatment algorithm in pulmonary arterial hypertension. J Am Coll Cardiol 2009; 54: 1 Suppl., S78-S84.

23 Mukerjee D, St George D, Coleiro B, et al. Prevalence and out come in systemic sclerosis associated pulmonary arterial hypertension: application of a registry approach. Ann Rheum Dis 2003; 62: 1088-1093.

24 Dorfmuller P, Humbert M, Perros F, et al. Fibrous remodeling of the pulmonary venous system in pulmonary arterial hypertension associated with connective tissue diseases. Hum Pathol 2007; 38: 893-902

25 Channick RN, Simonneau G, Sitbon O, et al. Effects of the dual endothelin-receptor antagonist bosentan in patients with pulmonary 
hypertension: a randomised placebo-controlled study. Lancet 2001; 358: 1119-1123.

26 Rubin LJ, Badesch DB, Barst RJ, et al. Bosentan therapy for pulmonary arterial hypertension. N Engl J Med 2002; 346: 896-903.

27 Kabunga P, Handler C, Das C, et al. Treatment of early systemic sclerosis associated pulmonary arterial hypertension with endothelin receptor antagonists improves survival. Eur Respir J 2007; 30: Suppl. 51, 250 .

28 Hachulla E, Gressin V, Guillevin L, et al. Early detection of pulmonary arterial hypertension in systemic sclerosis: a French nationwide prospective multicenter study. Arthritis Rheum 2005; 52: 3792-3800.

29 Denton $\mathrm{CP}$, Humbert M, Rubin L, et al. Bosentan treatment for pulmonary arterial hypertension related to connective tissue disease: a subgroup analysis of the pivotal clinical trials and their open-label extensions. Ann Rheum Dis 2006; 65: 1336-1340.

30 Denton CP, Pope JE, Peter HH, et al. Long-term effects of bosentan on quality of life, survival, safety and tolerability in pulmonary arterial hypertension related to connective tissue diseases. Ann Rheum Dis 2008; 67: 1222-1228.

31 Phung S, Strange G, Chung LP, et al. Prevalence of pulmonary arterial hypertension in an Australian scleroderma population: screening allows for earlier diagnosis. Intern Med J 2009; 39: 682-691.

32 Humbert M, Sitbon O, Chaouat A, et al. Pulmonary arterial hypertension in France: results from a national registry. Am J Respir Crit Care Med 2006; 173: 1023-1030.

33 Hachulla E, Carpentier P, Gressin V, et al. Risk factors for death and the 3-year survival of patients with systemic sclerosis: the French ItinérAIR-Sclérodermie study. Rheumatology 2009; 48: 304-308.

34 Badesch DB, Hill NS, Burgess G, et al. Sildenafil for pulmonary arterial hypertension associated with connective tissue disease. J Rheumatol 2007; 34: 2417-2422.
35 Girgis R, McLaughlin V, Hill N, et al. Sitaxsentan improves 6MW and hemodynamics in patients with pulmonary arterial hypertension (PAH) related to connective tissue disease (CTD). Ann Rheum Dis 2005; 64: Suppl. III, 283.

36 Montani D, O'Callaghan S, Jaïs X, et al. Implementing the ESC/ ERS pulmonary hypertension guidelines: real-life cases from a national referral centre. Eur Respir Rev 2009; 18: 272-290.

37 Launay D, Sitbon O, Le Pavec J, et al. Long-term outcome of systemic sclerosis-associated pulmonary arterial hypertension treated with bosentan as first-line monotherapy followed or not by the addition of prostanoids or sildenafil. Rheumatology 2010; 49: 490-500.

38 Galiè N, Negro L, Simonneau G. The use of combination therapy in pulmonary arterial hypertension: new developments. Eur Respir Rev 2009; 18: 148-153.

39 Galiè N, Brundage BH, Ghofrani HA, et al. Pulmonary Arterial Hypertension and Response to Tadalafil (PHIRST) Study Group. Tadalafil therapy for pulmonary arterial hypertension. Circulation 2009; 119: 2894-2903.

40 McLaughlin VV, Oudiz RJ, Frost A, et al. Randomized study of adding inhaled iloprost to existing bosentan in pulmonary arterial hypertension. Am J Respir Crit Care Med 2006; 174: 1257-1263.

41 Simonneau G, Rubin LJ, Galiè N, et al. Addition of sildenafil to long-term intravenous epoprostenol therapy in patients with pulmonary arterial hypertension: a randomized trial. Ann Intern Med 2008; 149: 521-530.

42 Galiè N, Manes A, Negro L, et al. A meta-analysis of randomized controlled trials in pulmonary arterial hypertension. Eur Heart $J$ 2009; 30: 394-403.

43 Williams $\mathrm{MH}$, Das C, Handler CE, et al. Systemic sclerosis associated pulmonary hypertension: improved survival in the current era. Heart 2006; 92: 926-932. 